\title{
Sibling curves and complex roots 1: looking back
}

Ansie Harding ${ }^{\mathrm{a}}$; Johann Engelbrecht ${ }^{\mathrm{a}}$

${ }^{a}$ Department of Mathematics and Applied Mathematics, University of Pretoria, South Africa

\section{Abstract}

This paper, the first of a two-part article, follows the trail in history of the development of a graphical representation of the complex roots of a function. Root calculation and root representation are traced through millennia, including the development of the notion of complex numbers and subsequent graphical representation thereof. The concepts of the Cartesian and Argand planes prove to be central to the theme. We specifically pause to look at efforts of representing complex roots of a function on the real plane, first, by superimposing the Argand plane onto the Cartesian plane, and secondly, by keeping the planes side by side and moving between the two, and thirdly, by taking the modulus of the function value and hence eliminating one dimension to enable drawing of the complex function as a surface in three dimensions.

\section{Historical perspective}

In this section on the trail in history of the millennia long quest for finding roots of functions, we made extensive use of a number of sources, including [1-9].

\subsection{In the beginning}

The first known solution of a quadratic equation is the one given in the Berlin papyrus 6619, which is an ancient Egyptian papyrus document from the 19th dynasty ca 13001200BC. The papyrus contains ancient Egyptian mathematical and medical knowledge, including the first known documentation concerning pregnancy test procedures. 
This document contains a problem [10]

The area of a square of 100 is equal to that of two smaller squares, the side of one square is $3 / 4$ of the other. What are the sides of the two unknown squares?

This question suggests some knowledge of what would later be called the solution of a second degree equation by taking squares and square roots.

The Babylonians of Mesopotamia (presently Iraq) prepared the ground for the work of alKhwarizmi. They knew how to solve certain type of quadratics and some cubic equations, although it is not known if they were able to reduce the general cubic equation. Babylonian clay tablets date from 1800 to $1600 \mathrm{BC}$.

Later, the Greeks were able to solve a quadratic equation by geometric methods as demonstrated by Euclid in 300BC. The first evidence of the use of algebraic methods for this purpose is the work of Diophantos ( $c$ a 210-29) who managed to partially solve the quadratic equation, only interested in positive rational solutions to these problems. Equations that would lead to solutions that are negative or render irrational square roots were considered as useless [11]. History then moves to the Middle East and beyond where patches of knowledge on the topic started appearing. Indian mathematicians gave rules to solve some cubics similar to the well-known quadratic formula before 500BC [12]. The Hindu mathematician Aryabhata ( $c a 475-550)$ gave a rule for the sum of a geometric series that shows knowledge of quadratic equations [11, 12]. Early studies of equations by al-Khwarizmi ( $c a$ 800) only allowed positive real roots. In 1079 Omar Khayyam (1050-1123), a famous poet and the leading mathematician of his time, solved cubics geometrically by intersecting parabolas and circles. However, he incorrectly conjectured the nonexistence of a general solution formula for cubics.

So for the first two thousand years knowledge on finding solutions of equations developed slowly. 


\subsection{During the Renaissance}

History took an interesting turn in the early Renaissance period in Europe. A first step in the algebraic solution of cubics was taken by Scipione dal Ferro (1465-1526), in

Bologna, who solved the equation $x^{3}+m x=n$. Scipione dal Ferro found a formula to solve a cubic equation similar to the quadratic formula. Nothing more is known of his discovery other than that he imparted it to his pupil Antonio Fior before his death. At the time, it was the practice to keep discoveries secret in order to secure an advantage over rivals by proposing problems beyond their mathematical reach [5].

A second step was taken by Nicolo Fontana (1506-1557). When still a boy he was so badly cut by a French soldier that he had a speech problem, and was called Tartaglia the 'stammerer". He was too poor to go to school and acquired knowledge of mathematics by himself, and became a teacher of mathematics at a university, first in Verona and later in Venice. Tartaglia found a method in 1530 to solve $x^{3}+m x^{2}=n$ but kept it secret [6].

A public contest took place between Tartaglia and Fior in 1535, the rules being that each gave the other 30 problems with 40 or 50 days in which to solve them, the winner being the one to solve most. Tartaglia solved all Fior's problems in the space of 2 hours, for all the problems Fior had set were of the form $x^{3}+m x=n$ as he believed Tartaglia would be unable to solve this type. Tartaglia solved thirty problems while Fior solved no $[2,6,13]$.

Girolamo Cardano (1501-1576) heard about Tartaglia's victory in Milan. Cardano invited Tartaglia to visit him and, after much persuasion, Tartaglia gave him his solution of the cubic equation in the form of a poem. Cardano promised to keep it secret until Tartaglia had published it himself but he did not keep this promise and in 1545 he published Ars Magna, the first Latin treatise on algebra, into which Tartaglia's work was included [2, 3, 13]. The formula for solving a cubic equation is credited to Cardano. Tartaglia did not deal with complex solutions. 
Cardano was the one to introduce complex numbers for the first time (at that stage rejected as absurd) in his complete solution of cubics. Cardano was the first to realize that one could work with quantities more general than the real numbers. He was able to manipulate with his 'complex numbers' to obtain the right answer yet he did not fully understand his own mathematics [1].

The well-known physicist Richard Feynman, [1]4 relates an interesting episode he experienced while on a visit to Greece in 1980:

They were very upset when I said the development of the greatest importance to mathematics in Europe was the discovery by Tartaglia that you can solve a cubic equation: although it is of little use in itself, the discovery must have been psychologically wonderful. It therefore helped in the Renaissance, which was freeing man from the intimidation of the ancients. What the Greeks are learning in school is to be intimidated into thinking they have fallen so far below their ancestors.

Tartaglia's work was done more than 1000 years after the Greeks and showed to the Greeks that a modern man could do something no ancient Greeks could do [15].

\subsection{The Fundamental Theorem of Algebra}

The first claim that a polynomial with degree $n$ always has $n$ roots was made by a Flemish mathematician Albert Girard in 1629. Shortly afterwards, in 1637, René Descartes (1596-1650) gave his rule of signs to determine the number of positive roots of a given polynomial saying that one can 'imagine' $n$ roots for every equation of degree $n$, but these imagined roots do not correspond to any real quantity. This was a problem of the time since mathematicians believed that a polynomial equation of degree $n$ must have $n$ roots, but could not prove that the roots were of the form $a+b i$ with $a$ and $b$ real as we now know it.

In 1702, Leibniz believed that he had produced a counter-example for (what we now call) the Fundamental Theorem of Algebra when he asserted that $x^{4}+t^{4}$ could not be written as 
a product of two real quadratic factors. He did not realize that $\sqrt{ }$ can be written in the form $a+b i, a, b$ real. It was 40 years later when Euler showed that the counter-example was false.

D'Alembert in 1746 made the first serious attempt at a proof of the Fundamental Theorem of Algebra but his proof was flawed; it came down to him assuming that the theorem was true in order to prove it. Three other well-known mathematicians of the 18th century, Euler, Laplace, and Lagrange, each had their own attempts at proving the theorem, but unfortunately none of them was able to produce a complete proof. Euler (1742) could prove that every real polynomial of degree $n,(n \leq 6)$ had exactly $n$ complex roots and later (in 1749) he partially succeeded in proving the general case for real polynomials. Some of the gaps in his proof was filled by Lagrange in 1772 using his knowledge of permutations of roots, but he was still assuming existence of roots of some kind in order to deduce that the roots were real and complex and had the form $a+b i, a, b$ real. Lagrange, in 1770, proved the important result that polynomials of degree five or more cannot be solved by the algebraic methods used for quadratics, cubics, and quartics.

Gauss is often credited with the first proof of the Fundamental Theorem of Algebra. In 1799 he presented his first proof and also his objections to earlier proofs. He was the first to spot the fundamental flaws in the earlier proofs, but ironically his 1799 proof also had some gaps. Gauss would later publish three further proofs of the theorem. Improving on D'Alembert's earlier proof, the Swiss mathematician Jean Robert Argand published a very simple proof of the theorem in 1814. Even though Argand is commonly credited with establishing the complex argument, he may have actually obtained the idea from another mathematician, Wessel. Wessel had the idea for the complex argument a few years ahead of Argand, however Wessel was born in Norway, and Scandinavian mathematicians were not that visible at the time [4].

In 1849 Gauss produced his fourth proof, which turned out to be his crowning achievement with respect to the fundamental theorem. It was the first proof that allowed 
the polynomials to have complex coefficients. Interestingly, Gauss believed that there existed a whole hierarchy of imaginary quantities of which complex numbers were the simplest. He called them a shadow of shadows.

Argand's proof was only an existence proof and it did not provide for the roots to be constructed. Weierstrass made a start towards a constructive proof in 1859 but it was not until 1940 that a constructive variant of the Argand proof was given by the German mathematician Hellmuth Kneser.

Formulas for the roots of polynomials up to a degree of 2 have been known since long ago (as discussed above) and up to a degree of 4 since the work of Cardano and Tartaglia in the 16th century. But formulas for degree 5 eluded researchers. In 1829, Abel proved the striking result that there can be no general formula (involving only the arithmetical operations and radicals) for the roots of a polynomial of degree 5 or greater in terms of its coefficients (the Abel-Ruffini theorem).

Emil Artin (1938) implemented the use of field theory to develop the modern theory of algebraic equations and in 1957 Arnold using results of Kolmogorov showed that it is possible to express the roots of the reduced 7 th degree polynomial in continuous functions of two variables, answering Hilbert's 13th problem in the negative. In 1991 Dummit and (independently) Kobayashi and Nakagawa gave methods for finding the roots of a general solvable quintic in radicals. Galois developed techniques for determining whether a given equation could be solved by radicals which gave rise to the field of Galois theory providing a detailed analysis of relationships between roots of polynomials. These techniques were first applied to finding a general criterion for determining whether any given quintic is solvable by Glashan, Young and Runge in 1885 (Wikipedia). Some fifth degree equations can be solved by factorising into radicals. Others cannot be factorized and solved in this manner. 


\subsection{Mechanical devices and computers}

An altogether different strand of development in finding roots is the construction of

mechanical devices for the purpose. The first of these machines for solving polynomial equations was built in 1770 by John Rowning (1699-1771). It was only practical for quadratics. In 1895 Quevedo built a machine for the mechanical calculation of the real and complex roots of a trinomial equation. Scientists at Bell Labs built the Isograph in 1935, a precision instrument that calculated roots of polynomials up to degree 15.

Presently, (with computer algebra as the one major exception), the study of higher order polynomial equations does not play such an important role in mathematics and computational mathematics. Software packages offer solutions at the press of a button and so eliminate the user from the need of knowing the intricacies of roots finding formulae and methods. These packages mostly make use of finding (approximate) roots by means of iterative algorithms of which there are many.

\subsection{The Cartesian plane and the Argand plane}

At this moment in our narration we turn back in time to the era of Descartes, the year 1637 to be precise, and pick up another strand that will help us complete our historical background for this study. Descartes, in his Discourse, gave a way to link an algebraic formula for a function to a picture of the function. The function is represented as a sketched curve on what nowadays is referred to as the Cartesian plane, a plane defined by two perpendicular axes with domain values on the horizontal axis and range values on the vertical axis. With the aid of this new tool features of functions such as turning points became visual and roots could be seen as points where the curve intersects the horizontal axis. Almost 200 years later, in 1814, Argand gave geometrical significance to complex numbers by interpreting $i$ as a rotation of the real line through $90^{\circ}$. The plane for representing complex numbers is referred to as the Argand plane.

Thus we came to have two planes, the Cartesian plane that can be used for showing the graph of a function and where its real roots lie and the Argand plane that gives a visual representation of complex numbers. The problem with these two distinct planes, a 
problem that is crucial to this study, is the following: If a function has complex roots, we can show these roots on the Argand plane but cannot see where they are on the graph of the function in the Cartesian plane.

\section{Reading complex roots from a graph}

Finding ways of reading the complex roots of a function from a sketch on the Cartesian plane has been the topic of many a paper. We set out to follow a main line of thought and in the process encountered many byways; various attempts by authors to visualise roots that are not considered as part of the main line of development [16-25, 27].

\subsection{Superimposing the Argand plane onto the Cartesian plane}

A frequently encountered approach to reading complex roots from a graph is to find a point $(x, y)$, related, in some way, to the root of a graph in the Cartesian plane, and then to interpret it as a complex number $x+i y$. This approach effectively means superimposing the Argand plane onto the Cartesian plane.

An example: For a quadratic function with two complex roots such as in figure 1, flip the curve vertically around its apex. The new curve cuts the x-axis twice. Take the midpoint of these two real roots as the centre of a circle with diameter the distance between the roots and draw the circle. Now pretend you are on the Argand plane. The two points on the circle vertically above and below the midpoint are the two complex roots of the original function. Why this is so follows easily from the fact that the curve and the inverted curve $f(x)=-(x-\alpha)^{2}+\beta^{2}$ has roots $\alpha \pm \beta$. 


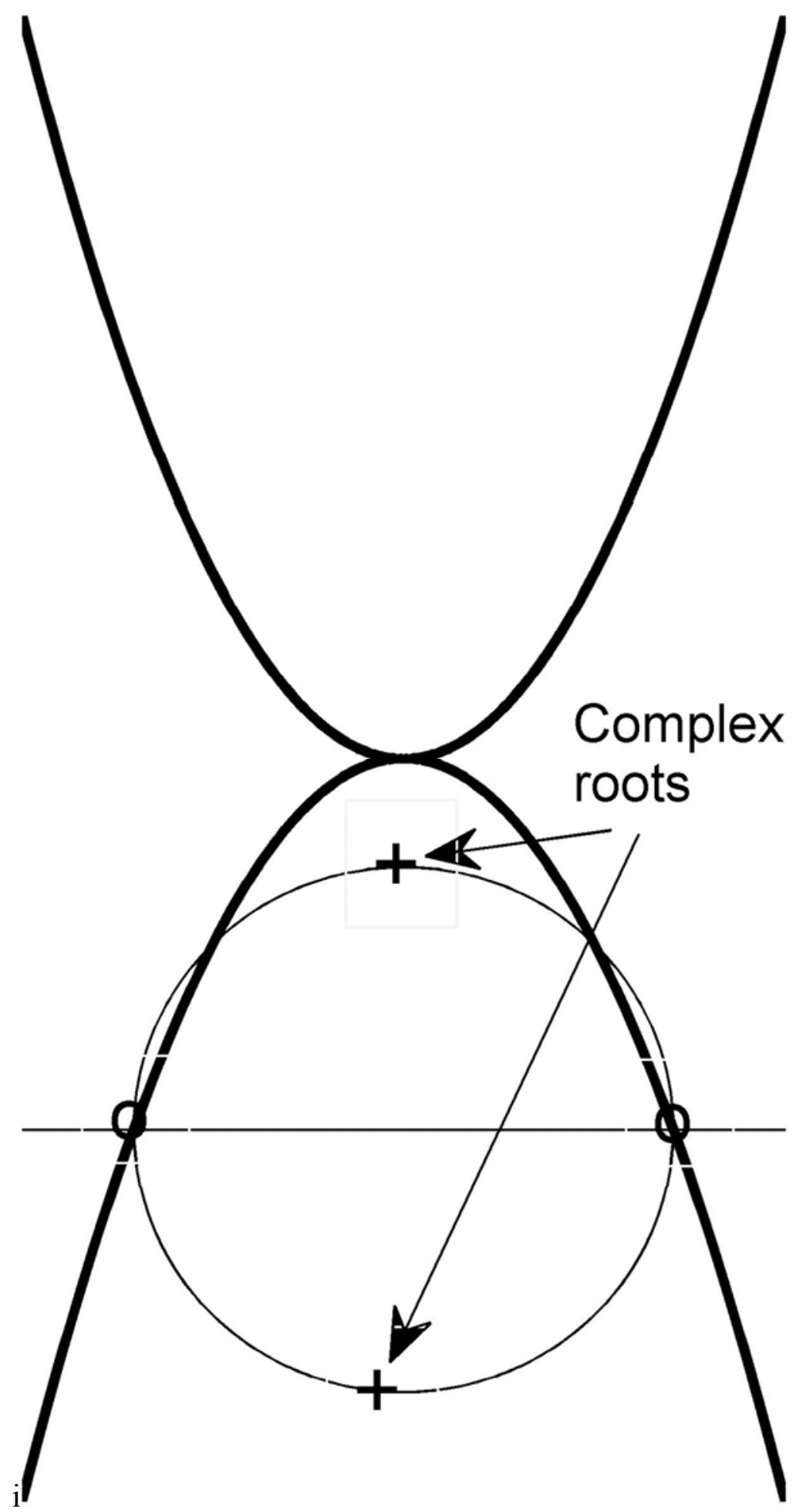

Figure 1. Superimposing the Argand plane onto the Cartesian plane. 
This simple construction originates from ideas in a relatively recent paper by Norton and Lotto [27] and is but one in a chain of efforts that further colours in the background of this paper. We follow this trail through the previous century when these ideas were developed, in order to reach a position to expand from.

The story starts in the early 1900s with papers by Gleason [28], Irwin and Wright [29] and Crawley [19] who all offer the same conclusions regarding a quadratic and a cubic polynomial.

For a quadratic function, shown in figure 2 , with two complex roots $\alpha \pm i \beta$ the minimum point on the graph has coordinates $\left(\alpha, \beta^{2}\right)$. So the abscissa of the minimum point is the real part of the roots and the ordinate is the square of the imaginary part.

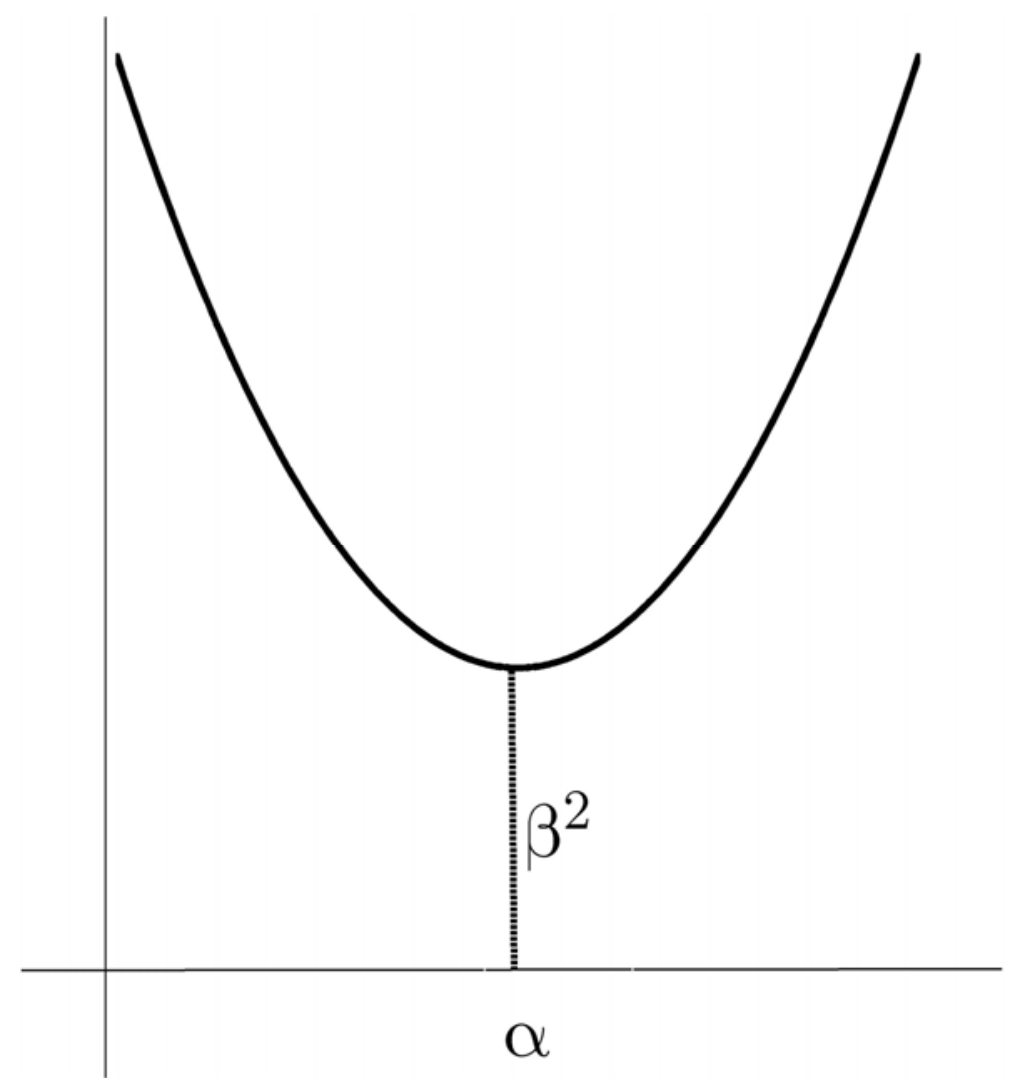

Figure 2. Reading complex roots from the graph of a quadratic function. 
One can also read $\alpha$ and $\beta$ directly from the graph by adding the line $y=2 \beta^{2}$. The abscissa of the points of interception with the graph are $\alpha \pm \beta$ [30].

For a cubic function, shown in figure 3, with one real root $x=p$ and two complex roots $x=\alpha \pm i \beta$, the line through the $x$-intercept $\mathrm{R}$ and tangent to the curve at $\mathrm{T}$, has a slope of $\beta^{2}$ and Thas abscissa $\alpha$.

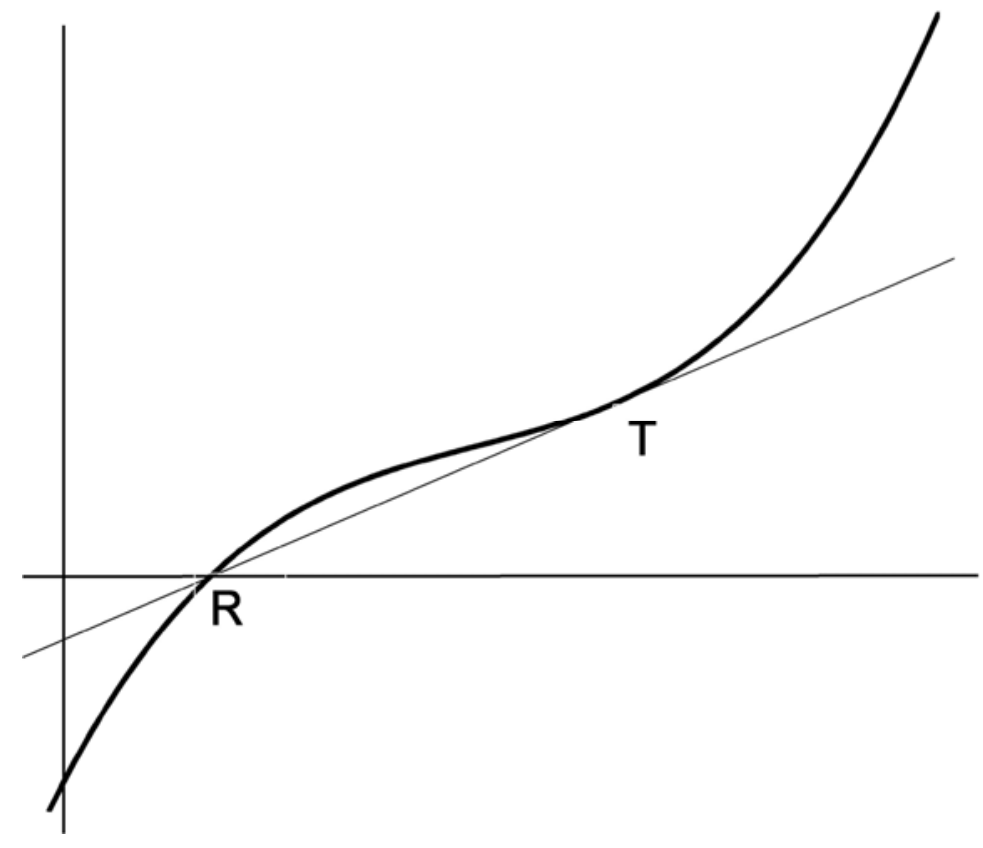

Figure 3. Reading complex roots from the graph of a cubic function.

This approach has also been expanded to the quartic cas [31, 32]. Then, in 1941 Gehman [33] combined all cases under the umbrella of a single theorem covering the cubic case and extending it to the quartic case and higher. The theorem is illustrated in figure 4 by a sketch of the quartic case: 


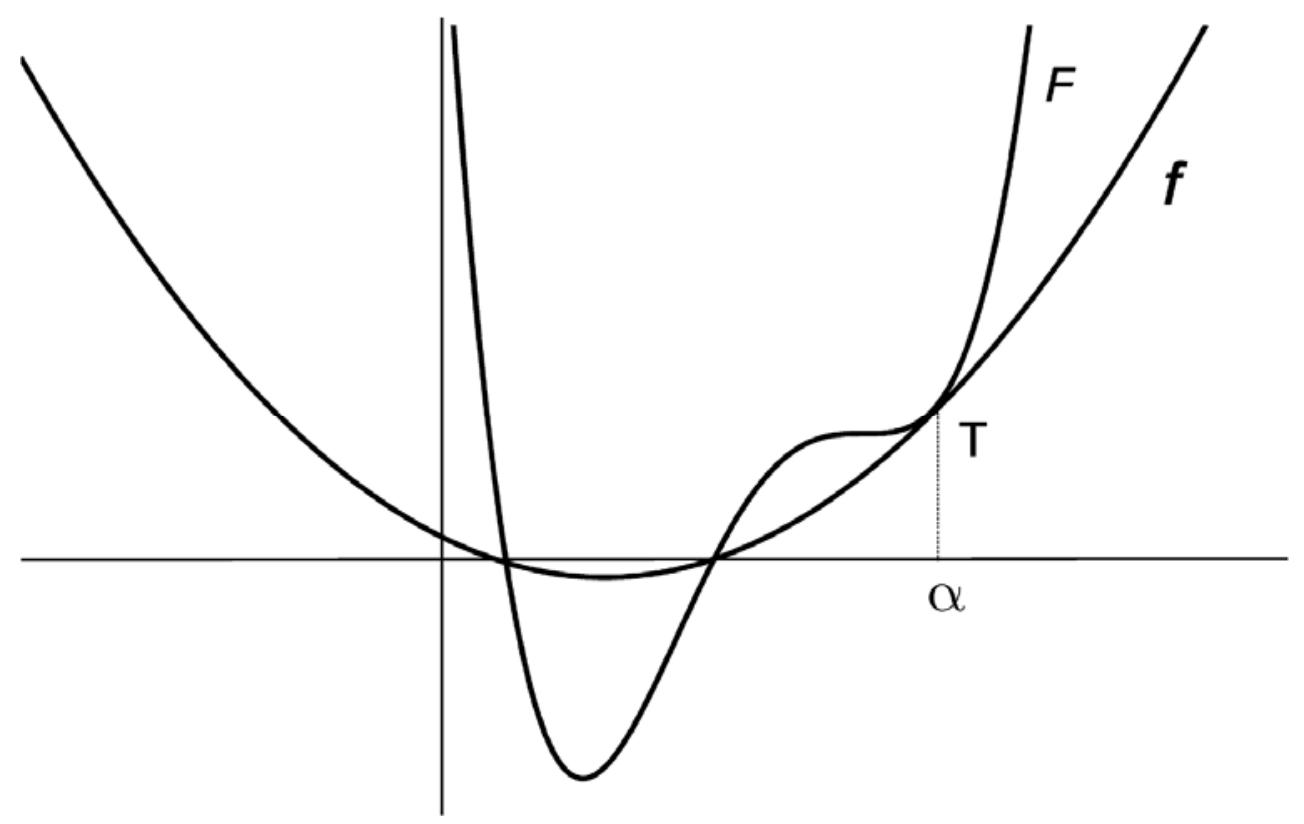

Figure 4. The quartic case for reading complex roots from the graph of a function.

For a polynomial of degree $n \geq 2$ with complex roots $\alpha \pm i \beta$ let $F(x)=\left(x^{2}-2 \alpha x+\alpha^{2}+\beta^{2}\right) f(x)$ where $f(x)$ is a polynomial of degree $n-2$ with real coefficients. There is a unique curve in the family $y=m f(x)$ which intersects the curve $y=F(x)$ on the $x$-axis in the $n$-2 real or complex points whose abscissa satisfy the equation $f(x)=0$ and is tangent to the curve $y=F(x)$ at a point in the real plane point $T$, having the property that the derivatives of the two functions at the point are equal from the first to the $k$ th where $k$ is the order of the highest ordered non-vanishing derivative at the point $T$. The abscissa of the tangency is $\alpha$ and the slope of the tangent is $\beta^{2}$ [33].

The formulation of the this theorem exhausted the particular train of thought except for one other paper by Yanosik [34] in which he discusses the quartic case, in more detail, for functions with various combinations of real and complex roots and manages to read the complex roots graphically by means of further constructions.

\subsection{Moving between the Cartesian and Argand planes}

It was time for a new approach and this came in 1937 in a paper by Ward [35] where he separated the Cartesian and Argand planes and found connections between them. The 
work in this paper relates directly to the current study and it is necessary to investigate with a little more detail.

It is easy to show that the general cubic with real coefficients may be reduced by a linear transformation to

$$
f(x)=x^{3}+p x+t
$$

If $u+i v$ is a complex root of the cubic and is substituted into (1), both the real and imaginary parts vanish. So

$$
v\left(-v^{2}+3 u^{2}+p\right)=0
$$

Since $v \neq 0$ then

$$
-v^{2}+3 u^{2}+p=0
$$

which is a hyperbola in the complex plane. This hyperbola is the locus of the complex roots of (1) with $t$ as a parameter.

In the graph of $y=-x^{3}-p x$, the abscissas of its intersections with the line $y=t$ give the real roots of (1). If $r$ is the only real root, the real part of the imaginary roots is $-r / 2$ because the sum of the roots of (1) is zero. Hence, the imaginary roots will be the intersection of the line $u=-r / 2$ and the hyperbola as shown in figure 5 . 


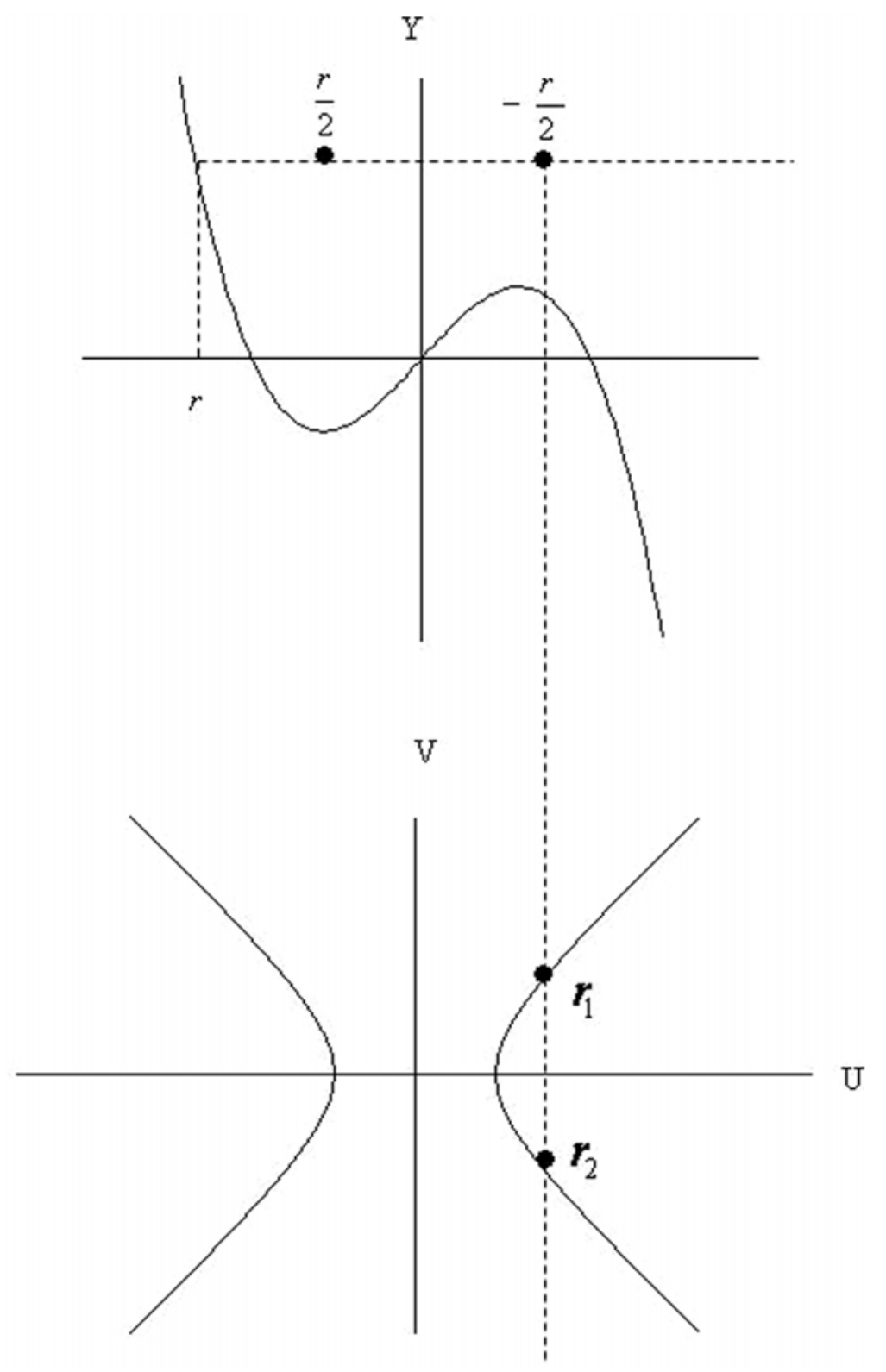

Figure 5. Moving between the Cartesian and Argand planes.

A geometrical representation simplifies the root finding.

Place the $u v$-plane below the $x y$-plane so that the $v$-axis and the $y$-axes are collinear and draw sketches on the same scale as in figure 5. If there is one real root, $y=t$ will cut the cubic in $x=r$. Find the midpoint $(r / 2, t)$ and the reflected point $P(-r / 2, t)$. A vertical line through $x=-r / 2$ will cut the hyperbola in the complex roots $r_{1}$ and $r_{2}$ of (1). 
If $p$ is positive, the transverse axis of the hyperbola is along the $u$-axis and the two complex roots are on different branches of the hyperbola.

A similar procedure can be followed for the quartic polynomial. In this case the locus of the complex roots of the reduced quartic equation

$$
x^{4}+p x^{2}+q x+t=0
$$

is given by

$$
4 u v^{3}=4 u^{3}+p u+q
$$

The graph of (5) has three fixed asymptotes $u=0, u=v, u=-v$ which are independent of $p$ and $q$ and hence there are three distinct branches of the graph.

\subsection{Combining the Cartesian and Argand planes}

From superimposing the Argand plane onto the Cartesian plane to separating the planes and moving from one to the other, the next step in the development was to combine the two planes to get a three dimensional view. The Argand plane is placed perpendicular to the Cartesian plane so that the $x$ - and $y$-axes remain the absis (horizontal) and ordinate (vertical) axes in the real plane, respectively, and the third (horizontal) axis is use to represent the complex parts of a number, call it the $w$-axis. The three planes are the $x y$ plane, the $x w$-plane and the $y w$ - plane.

The result is that for a function $f$ that maps complex numbers onto a real numbers the $x w$ plane is where the domain is hosted and the range will be found on the $y$-axis. This approach coincided with the advent of the computer era in the 1970s, perhaps because visualization became easier.

Long [36, 37] and Long and Hern [38] make use of this three-dimensional set of axes to have a representation of a function in terms of what they call modulus surfaces. With each point $z$ in the complex plane associate the value of the modulus $|f(z)|$ of the polynomial $f$ at that point. The graph of the function $|f(z)|$ is called a modulus surface. As

an example the modulus surface of $f(z)=z^{2}+z+1$ is shown in figure 6 . From the figure the 
visual advantage of a modulus surface representation for graphically displaying the roots of a function is clear. Roots are found where the modulus surface has minimum points touching the $x w$-plane. Surfaces became easy to draw with the use of computer graphics. In addition, it enabled the authors to see how see how the zeros of a polynomial moved when a single coefficient is changed. In fact, the authors acknowledge that two of their main theorems were discovered via computer experimentation.

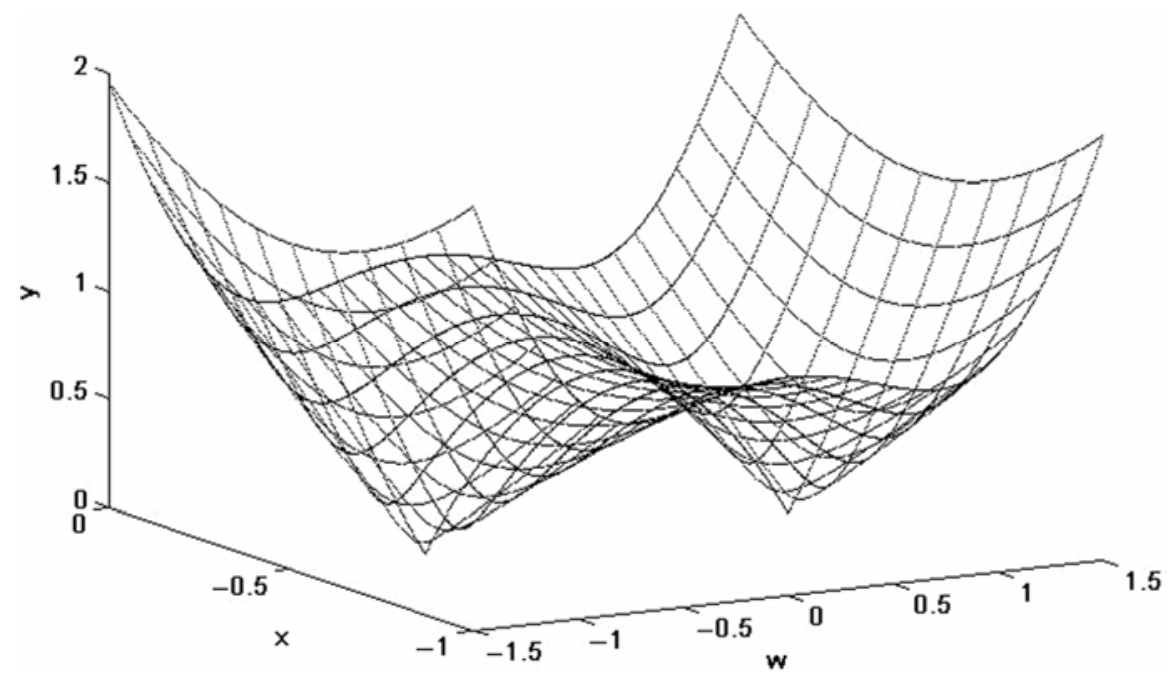

Figure 6. The modulus surface of $f(z)=z^{2}+z+1$.

Computer graphics were also the inspiration behind the work of Velleman [39] who makes use of colour coding to give a visual representation of a function mapping complex numbers onto complex values.

Although both latter representations are visual and illustrative, we feel that both these approaches simply offer a compromise for not being able to fully visualize graphs of functions that map complex numbers onto complex numbers. We feel that the wrong avenue is pursued. The fact is that in our quest for looking for roots we have only ever felt the need to consider those complex numbers that map onto real numbers, not onto complex numbers. For a function that maps complex numbers onto complex numbers, we are only interested in that part of the domain that maps onto real values. This is an important notion. 


\section{References}

- 1. O'Connor, JJ and Robertson, EF (2006) The fundamental theorem of algebra. The MacTutor History of Mathematics archive - Retrieved March 2007 from www-groups.dcs.st-nd.ac.uk/history/HistTopics/Fund_theorem_of_algebra.html

- 2. O'Connor, JJ and Robertson, EF (2006) Quadratic, cubic and quartic equations. The MacTutor History of Mathematics archive — Retrieved March 2007 from www-groups.dcs.st-and.ac.uk/history/HistTopics/Quadratic_etc_equations.html

- 3. O'Connor, JJ and Robertson, EF (2006) Tartaglia versus Cardan. The MacTutor History of Mathematics archive - Retrieved March 2007 from wwwgroups.des.st-and.ac.uk/ history/HistTopics/Tartaglia_v_Cardan.html

- 4. Throop, A. (2005) The Fundamental Theorem of Algebra Kennesaw State University , Kennesaw, GA — Student project, Retrieved April 2007 from http://ksuweb.kennesaw.edu/ jderado/Students/ProofFTA.pdf

- 5. Guilbeau, L. (1930) The history of the solution of the cubic equation. Mathematics News Letter 5:4, pp. 8-12.

- 6. Struik, DJ (1962) A Concise History of Mathematics G Bell \& Sons, London

- 7. Passagen (Current) — Internet website. Retrieved April 2007 from http://hem.passagen.se/ceem

- 8. Wikipedia. (Current) Various articles on mathematics and the history of mathematics - Retrieved February 2007 from http://en.wikipedia.org

- 9. Wolfram Mathworld. (Current) Quadratic equation - Retrieved April 2007 from http://mathworld.wolfram.com/QuadraticEquation.html

- 10. Gillings, RJ (1982) Mathematics in the time of the Pharaohs p. 161. Dover, New York

- 11. Smith, D. (1951) History of Mathematics 1 , Dover, New York

- 12. Smith, D. (1953) History of Mathematics 2 , Dover, New York

- 13. Nordgaard, MA (1938) Sidelights on the Cardan-Tartaglia controversy. National Mathematics Magazine 12:7 , pp. 327-346.

- 14. Feynman, RP (1988) What Do You Care What Other People Think? W. W. Norton, New York 
- 15. Griffiths, HB and Hirst, AE (1994) Cubic equations, or where did the examination question come from. American Mathematical Monthly 101:2, pp. 151-161.

- 16. Math, Ask dr (1994) Complex roots. Internet discussion on The Math Forum@Drexel — Retrieved March 2007 from http://mathforum.org/library/drmath/view/53808.html

- 17. Ballantine, JP (1920) A graphic solution of the cubic equation. The American Mathematical Monthly 27:5 , p. 204.

- 18. Braden, B. (1985) Picturing functions of a complex variable. The College Mathematics Journal 16:1 , pp. 63-72.

- 19. Crawley, ES (1918) Relating to the graph of a cubic equation having complex roots. American Mathematical Monthly 25:6, pp. 268-269.

- 20. Curtis, HB (1938) A graphical solution of the cubic. National Mathematics Magazine 12:7 , pp. 325-326.

- 21. Faucette, WM (1996) A geometric interpretation of the solution of the general quartic polynomial. American Mathematical Monthly 103:1 , pp. 51-57.

- 22. Gomez-Calderon, J. and Wells, DM (1996) Why polynomials have roots. The College Mathematics Journal 27:2 , pp. 90-94.

- 23. Rees, EL (1922) Graphical discussion of the roots of a quartic equation. American Mathematical Monthly 29:2, pp. 51-55.

- 24. Running, TR (1921) Graphical solutions of quadratic, cubic, and biquadratic equations. The American Mathematical Monthly 28:11/12, pp. 415-423.

- 25. Travers, R. and Kim, D. (1982) Those elusive imaginary zeroes. Mathematics Teacher 75 , pp. 62-64.

- 26. Teacher 2 Teacher (2003) The geometry of the complex roots of graphs. Internet discussion on Mathforum - Retrieved April 2007 from http://mathforum.org/t2t/message.taco?thread=12605\&message $=1$

- 27. Norton, A. and Lotto, B. (1984) Complex roots made visible. The College Mathematics Journal 15:3 , pp. 248-249.

- 28. Gleason, RE (1910) A simple method for graphically obtaining the complex roots of a cubic equation. The Annals of Mathematics 2nd Series 11:3, pp. 95-96. 
- 29. Irwin, F. and Wright, HM (1917) Some properties of polynomial curves. The Annals of Mathematics 19:2, pp. 152-158.

- 30 . Wessels, SFG (1981) The complex roots of a quadratic from its graph. Mathematical Gazette 65 , p. p 39.

- 31. Henriquez, G. (1935) The graphical interpretation of the complex roots of cubic equations. American Mathematical Monthly 42:6, pp. 383-384.

- 32. Yanosik, GA (1936) A graphical solution for the complex roots of a cubic. National Mathematics Magazine 10:4, pp. 139-140.

- 33. Gehman, HM (1941) Complex roots of a polynomial equation. American Mathematical Monthly 48:4, pp. 237-239.

- 34. Yanosik, GA (1943) Graphical solutions for complex roots of quadratics, cubics and quartics. National Mathematics Magazine 17:4, pp. 147-150.

- 35. Ward, JA (1937) Graphical representation of complex roots. National Mathematics Magazine 11:7, pp. 297-303.

- 36. Long, CA (1971) A note on the geometry of zeros of polynomials. Mathematics Magazine 44:3, pp. 157-159.

- 37. Long, CA (1972) The quadratic polynomial and its zeros. The Two-Year College Mathematics Journal 3:1 , pp. 23-29.

- 38. Long, C. and Hern, T. (1989) Graphing the complex zeros of polynomials using modulus surfaces. The College Mathematics Journal 20:2 , pp. 98-105.

- 39. Velleman, DJ The Fundamental Theorem of Algebra: A visual approach Unpublished paper. Retrieved March 2007 from http://www.cs.amherst.edu/ djv/FTAp.pdf 